\title{
Statin Treatment May Play a Key Role in the Prevention of Dementia: A Review of the Literature
}

\author{
By Chiara Bandinelli, Giovanni Maria Puddu, Luca Spinardi, \\ Giampaolo Bianchi \& Marco Zoli
}

Introduction- Dementiais characterized by cognitive decline and deterioration of daily function, often with behavioral disturbances. Degenerative dementia includes many subgroups like Alzheimer's disease (AD), frontotemporal dementia, etc, and nondegenerative dementia mainly encompasses vascular dementia, traumatic brain dementia, and so on. Alzheimer's diseaseis the mostcommon dementia in the elderly, accounting for $60 \%$ of cases (1). There isn't specific therapy for degenerative dementia, only symptomatic treatment.

GJMR-A Classification: NLMC Code: WM 220

Strictly as per the compliance and regulations of:

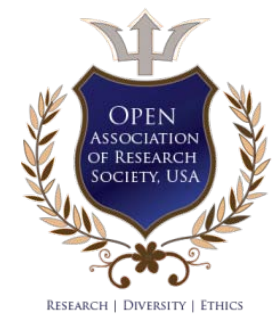

(C) 2020. Chiara Bandinelli, Giovanni Maria Puddu, Luca Spinardi, Giampaolo Bianchi \& Marco Zoli. This is a research/review paper, distributed under the terms of the Creative Commons Attribution-Noncommercial 3.0 Unported License http://creativecommons.org/licenses/by-nc/3.0/), permitting all non-commercial use, distribution, and reproduction in any medium, provided the original work is properly cited. 


\section{Statin Treatment May Play a Key Role in the Prevention of Dementia: A Review of the Literature}

Chiara Bandinelli ${ }^{\alpha}$, Giovanni Maria Puddu ${ }^{\sigma}$, Luca Spinardi ${ }^{\rho}$, Giampaolo Bianchi ${ }^{\omega}$ \& Marco Zoli ${ }^{*}$

\section{INTRODUCTION}

$\mathrm{D}$ ementia is characterized by cognitive decline and deterioration of daily function, often with behavioral disturbances. Degenerative dementia includes many subgroups like Alzheimer's disease (AD), frontotemporal dementia, etc, and nondegenerative dementia mainly encompasses vascular dementia, traumatic brain dementia, and so on. Alzheimer's diseaseis the mostcommon dementia in the elderly, accounting for $60 \%$ of cases (1). Thereisn't specific therapy for degenerative dementia, only symptomatic treatment.

Table 1: Causes of dementia

\section{Causes of dementia: classification}

-Alzheimer disease (60\%)

- Cerebrovascular disease (20\%): multi infarct dementia; subcortical vascular disease

-Neurodegenerative: Parkinson's disease; Huntington's corea; Pick's disease

-Infections: HIV; encephalitis; Creutzfeld-Jackob's disease

- Normal pressure hydrocephalus

-Nutritional: vitamine deficiencies (B12, folate, thiamine)

- Metabolic: thyroid disease, hepatic disease

- Inflammatory: chronic inflammation (vasculitis)

- Trauma

-Tumor

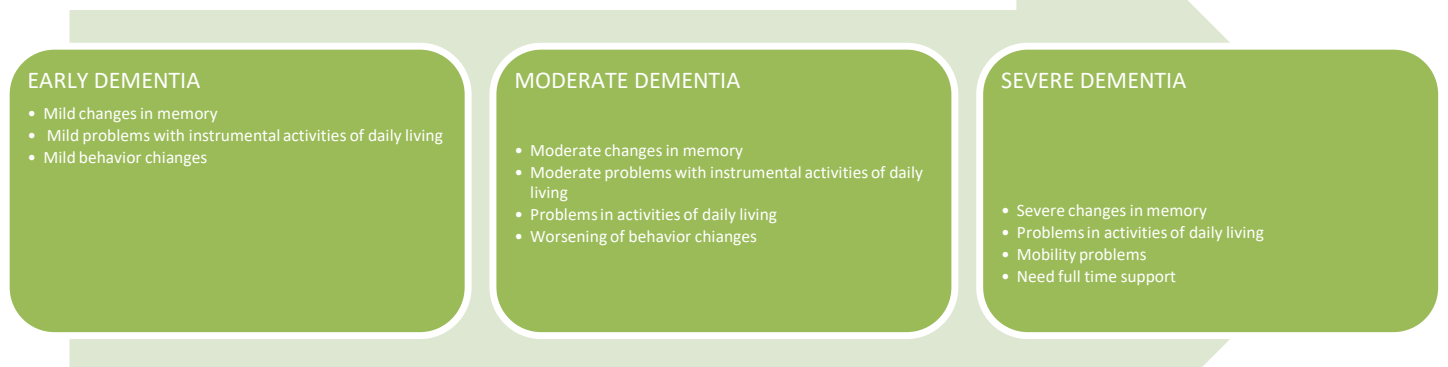

Figure 1: Dementia stages

Author a $\sigma \omega ¥:$ U.O. Stroke Unit Policlinico Sant’Orsola Bologna. e-mail: chiara.bandinelli2@studio.unibo.it Author p: U.O. Neuroradiologia Policlinico Sant'Orsola Bologna. 
There are different mechanisms that can explain the degeneration process, including inflammation, oxidative stress, circulation of blood to the brain, increased cerebral $\beta$-amyloidpeptides, hyperinsulinemia, brain insulin resistance, andt he formation of advancedglycation end- products (2).

In literature, numerous studies havefound the implication of statinsas protectors for brain degeneration and how statins use mightlower the risk of dementia (3).

Lipidaltering agents encompass classes of drugsthatinclude hydroxymethylglutaryl-CoA (HMG) (Coenzyme A) reductase inhibitors or statins, fibric acid derivatives, bile acid sequestrants, cholesterol absorption inhibitors, and nicotinic acid. These drugs stand out for the action and type of lipid-lowering. So the indications for a particular lipid-altering agent are conditioned by the kind of lipid abnormality (4).

Statins are the most commonly prescribed class of drugs to reduce cholesterol. Their action mode is the competitive inhibition of the active site of HMG-CoA reductase, the speed-limitingenzyme in the cholesterol biosynthesis pathway. The inhibition of this active site avoids substrate access, blocking the conversion of HMG-CoA to mevalonic acid. This mechanism reduce shepatic cholesterol synthesis, increasing the production of microsomal HMG- CoA reductase and the expression of cellsurface LDL receptor, which facilitates the clearance of LDL-c from the blood and a reduction in circulating LDL-c levels. Thanks to this mechanism, statins reduce cardiovascular and cerebrovascular morbidity in primary and secondary prevention. Statinsalso have further non-lipid-related pleiotropic effects, probablydue to the inhibition of the synthesis of isoprenoid intermediates of the mevalonate pathway. These mechanisms include improvements in endothelial function, stabilization of atheroscleroticplaques, antiinflammatory, immunomodulatory and antithrombotic effects, effects on bone metabolism, and reduced risk of dementia (5).

Table 2: Classification of statins by expected LDL reduction
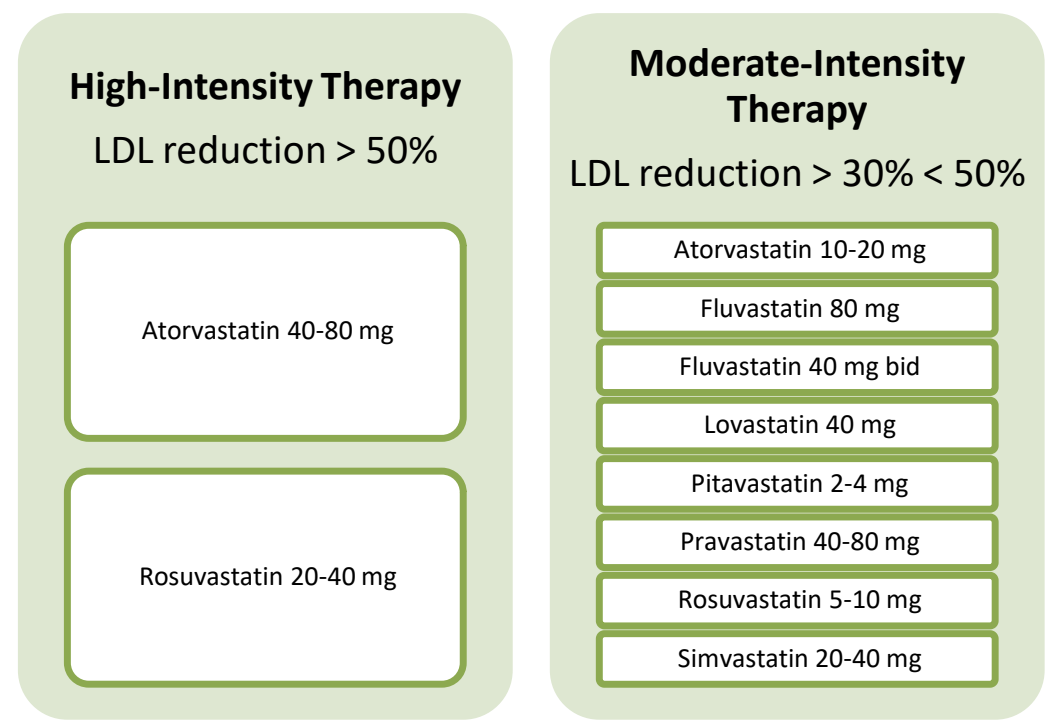

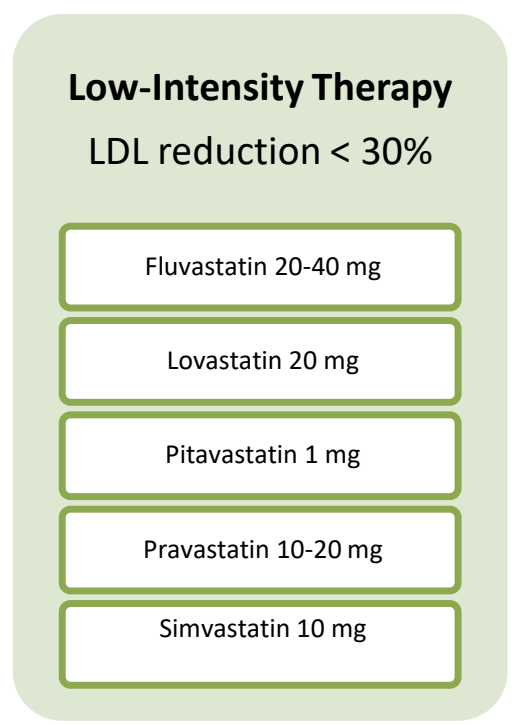

As regards dementia, cholesterol can be deposited in the brain, specifically in the hippocampus, causing the degradation of the amyloid precursor protein, which causes degeneration of neurons, resulting in $A D$. Statins, with their lipid-lowering effect, may reduce the formation of the b-amyloid peptide in the brain. In fact, they have an impact on the homeostasis of the nervous system cholesterol, inhibit the synthesis of cholesterol, lower the cholesterol level, and thus inhibit the beta metabolism of the amyloid precursor protein. Also, apoE4, a cholesterol transporter, is a risk factor and genetic marker of familiar $A D$ and contributes to b-amyloid deposition and the development of senile plaques. Statins inhibit apoE secretion and lower extracellular apoE levels, consequently preventing the formation of senile plaques and enhancing cognitive function. Moreover is known an age-related cognitive syndrome called cerebral small vessel disease (CSVD), which includes group of pathological processes with multiple causes that affects small cerebral vessels. Features associated with CSVD include white matter hyperintensities (WMH), lacunes, enlarged perivascular space (EPVS), and microbleeds observed through magnetic resonance imaging (MRI). CSVD can be asymptomatic; however, lesions can cause cognitive dysfunction, dementia, and mood disorders (6). Therefore measures to prevent CSVD are particularly crucial. CSVD is associated with vascular risk factors, including smoking, hypertension, hyperlipidemia, and aging. The association between 
statin therapy and CSVD is well documented in the elderly: numerous evidences show that statins may

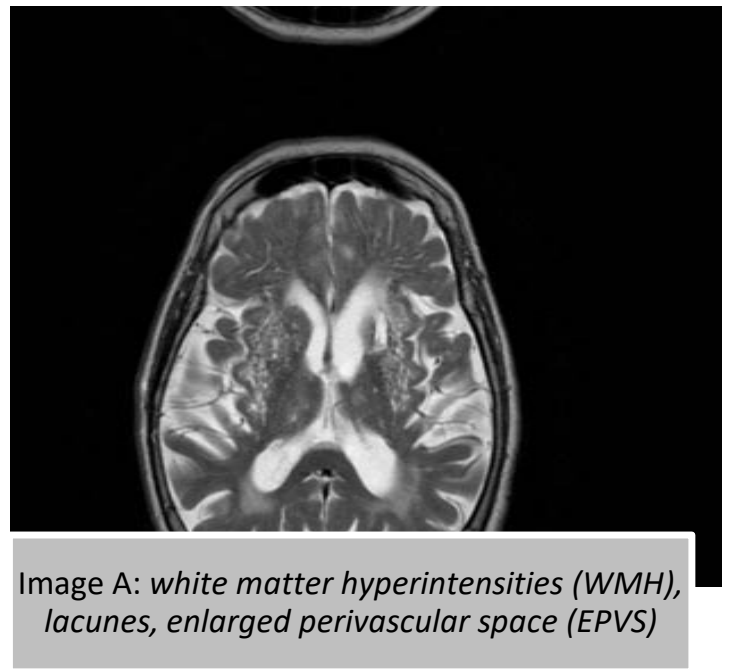

Figure 2: MRI brain sequences, T2 fast spin-echo(FSE) image A and T2* gradientecho (GRE)image B

\section{Discussion}

In the literature, there are several results about use and risk of dementia.

In November 2017, Medline, Embase, Web of Science, and the Cochrane Database identify the potential relationship between statins and dementia. Statins use was related to dementia risk decrement. Subgroup analysis demonstrates statins use was connected to Alzheimer's disease (AD) and non-AD dementia risk decrement (8).

In 2013 Chang CF, Liou YS, Lin TK et al. investigated the risk of new-onset dementia (NOD) in statin users compared to non-users. The risk of NOD was lower among statin users than non-users. The protective effect of statins for NOD seemed to be realted to high exposure to statins (9).

Cheng PY, Liu SK, Chen CL et al. found a duration-response relationship with long-term use of statin and dementia risk: one year of treatment of statins decreased by $9 \%$ the risk of dementia. The use of high dose statins for more than one year correlated with a lower risk of dementia than the use of low dose. On the contrary, fibrates or other lipid-lowering agents had no significant association with dementia risk (10). Even Hendrie $\mathrm{HC}$, Hake $\mathrm{A}$, Lane $\mathrm{K}$, et al. found that only consistent use of statin medications during years significantly reduces the risk of incident AD (22).

For mild cognitive impairment (MCl), Bettermann K, Arnold AM, Williamson $\mathrm{J}$ et al. studied statin drugs and the cognitive delay decline and dementia onset in individuals with and without mild cognitive impairment $(\mathrm{MCl})$ at baseline. They found that statins may slow the rate of cognitive decline and delay the onset of $A D$ and all-cause dementia in cognitively decrease major vascular events and alleviate the progression of CSVD and, consequently, dementia (7).

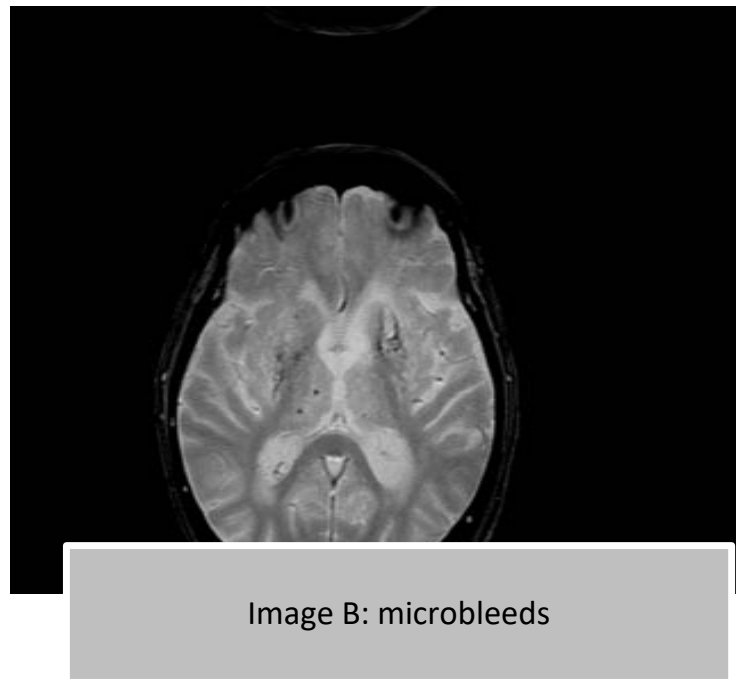

severe problem. In 2009 Horsdal HT, Olesen AV, Gasse $\mathrm{C}$ et al. examined the risk of admission with dementia related with utilization of statins in a case- control study in 4 Northern Danish counties. They found a reduced risk of hospitalization with dementia among users of statins (16).

In addiction, Reed B, Villeneuve S, Mack W et al, investigated the association between serum cholesterol levels and cerebral b-amyloid in the $A D$ process. The elevated cerebral b-amyloid level was

healthy elderly individuals. On the other hand, patients with $\mathrm{MCl}$ may not have a comparable effect from statin use (11).

Alzheimer's disease progression compared to late treatment, especially in $A D$ evolution in mild-to-moderate class (12).

After stratifying by gender, the risk of incident dementia was lower in female statin users than in male statin users. It were required higher potency and longer cumulative duration of statin use for reducing the risk of incident dementia in male patients than in female patients (13).

Several studies analyzed the relationship between the subclasses of statins and the prevention of dementia. Wu CK, Yang YH, Lin TT et al. found that the more potent statins (e.g., atorvastatin and rosuvastatin) seemed to be particularly effective in the prevention of dementia (14)

On the contrary, Wolozin B, Wang SW, Li NC et al. found an association with Simvastatin and a severe reduction in the incidence of dementia and Parkinson's disease. Atorvastatin is associated with a modest decrease in incident dementia (15).

The hospitalization in people with dementia is a 
associated with high cholesterol fractions suggesting a significant role for cholesterol in b-amyloid processing.

Cholesterol levels are modifiable with statin use. So, understanding the link between cholesterol levels and the b-amyloid deposition could potentially have an effect on retarding the pathologic cascade of $A D(17)$.

Analyzing patients with ApoE4 homozygotes, the incidence of $A D$ was significantly lower in statin users, and ApoE4/ApoE4-genotyped $A D$ patients treated with statins showed better cognitive function throughout 10-year follow-up (18).

Regarding CSVD, a cohort study made by Guo Y, Li Y, Liu X, et al (19) analyzed MRI features associated with CSVD. They found that white matter hyperintensities (WMH) volume, WMH-to-intracranial volume (ICV) ratio, lacunes, enlarged perivascular spaces (EPVS) and microbleeds were significantly lower in the statin group than the non-statin group.

In contrast, according to the Pravastatin in Elderly Individuals at Risk of Vascular Disease (PROSPER) trial (20), there's no evidence of benefit from statin treatment on prevention of dementia: PROSPER trial randomized patients age 70 to 82 years to pravastatin or placebo.

Patients had to perform cognitive tests at regular intervals to control memory and executive function. They have not found significant difference in cognitive function between the treatment and control groups after 42 months of follow - up (21).

\section{ili. Conclusions}

Dementia is a disease that afflicts many patients, and unfortunately, there is still no class of drugs that can treat this disease. Therefore, finding medicines that can prevent or delay the progression of the disease is crucial and that's what we set out to discover when we started this review.

Statins are widely prescribed drugs in the population for their protective role in cardio and cerebrovascular events. Accumulating evidence suggests that statins may play a decisive role in the prevention of dementia.

Several studies showed that the protective effect of statins might depend on the duration (years). and the high dose of the drug. In fact, lower dosages of the drug appear appear to have less action on disease progression as well as shorter duration.

The early statin use seems to be protective for the progression of Alzheimer's disease while later use may not affect this process.

Mild cognitive impairment $(\mathrm{MCl})$ is the stage between the cognitive decline of normal aging and the more severe decline of dementia. Our researches show that for $\mathrm{MCl}$, there is no evidence that statins give cognitive protection for the onset of dementia. Probably their protective effect emerges more when is already expressed the mental deterioration. Concerning the subclasses of statins, there are controversial opinions on their efficacy delaying the progression of dementia (for some, atorvastatin and rosuvastatin are the most effective; for others, it is simvastatin).

Although some subclasses are more effective or faster than others, all statins still appear to delay the progression of dementia.

Stratifying by gender, the risk of dementia for statin users is lower in females than the males. In male patients is required higher potency and longer cumulative duration of statin than the females. We could explain this process by pathophysiological differences between genders and different hormone-modulated pharmacologic or metabolic mechanisms. The hormonal treatment in postmenopausal women has been associated with the decrease in cholesterol levels, even if the association between hormonal therapy and dementia is still unexplained. So considering the differences between gender is very important in the clinical practice about the use of statin.

Hospitalization is a massive problem that affects many patients with dementia. During hospitalization, the patient worsens cognitive function, becomes disoriented, and may develop behavioral disturbances. Preventing hospitalization could be crucial to limiting the cognitive and behavioral complications of dementia. We found several studies that show that the risk of hospitalization in dementia patients is lower in statins users; this finding, therefore, provides some support to the hypothesis that statins may protect against the development of dementia.

Regarding the molecular pathogenesis of dementia, especially $A D$, reports have indicated that there is a molecular association between the senile degeneration process in dementia and serum cholesterol levels: evidences show a correlation between elevated cerebral $A B$ level and cholesterol fractions, suggesting a crucial role for cholesterol in $A B$ processing. Using lipid-altering agents like statins could, therefore reduce serum cholesterol levels and thus promote less deposition of beta-amyloid in the brain.

Overall our results suggest that the utilization of statins may benefit all $A D$ patients, especially those with an ApoE4 homozygotes genotype. In ApoE4 homozygotes, the incidence of $A D$ is significantly lower in statin users. For future research, it would be interesting to explore the most appropriate genotypes for statin use as a preventive therapy of AD.

Traditional vascular risk factors (like diabetes, hypercholesterolaemia, hypertension, and smoking) increase the risk for CSVD, which is a significant factor in the pathogenesis of dementia. Cholesterol- lowering medications, such as statins, may have a crucial role to prevent CSVD and consequently treat and anticipate the 
cognitive impairment. All in all, from the accurate review of the scientific literature, statins could have a protective role in the progression of dementia. Conversely, few studies have found no benefit from drug treatment compared to placebo. In any case, we found no aggravating effect of the statin on cognitive impairment.

We, therefore, concluded that statins might play a crucial role in the prevention of dementia. In the scientific field, their use could be an excellent resource for delaying the progression of cognitive decline. Given that dementia is the most common degenerative disease in the elderly, it is clear that its prevention can be crucial in the geriatric setting, and statins could play an essential role in this process.

\section{Bibliography}

1. Abraha I, Rimland JM, Trotta FM, et al. Systematic review of systematic reviews of nonpharmacological interventions to treat behavioural disturbances in older patients with dementia. The SENATOR-OnTop series [published correction appears in BMJ Open. 2017 Jul 17;7(7): e012759corr1]. BMJ Open. 2017;7(3):e012759. Published 2017 Mar 16. doi:10.1136/bmjopen-2016012759.

2. Campbell JM, Stephenson MD, de Courten B, et al. Metformin and Alzheimer's disease, dementia and cognitive impairment: a systematic review protocol. $\mathrm{JBI}$ Database System Rev Implement Rep. 2017;15(8):2055-2059. doi:10.11124/JBISRIR-2017003380.

3. Pan ML, Hsu CC, Chen YM, et al. Statin Use and the Risk of Dementia in Patients with Stroke: A Nationwide Population-Based Cohort Study. J Stroke Cerebrovasc Dis. 2018;27(11):3001-3007. doi:10.1016/j.jstrokecerebrovasdis.2018.06.036.

4. Robert S Rosenson, Mason W Freeman, Jane Givens. Statins: Actions, side effects, and administration.

5. Natalie C. Ward, Gerald F. Watts, Robert H. Eckel. Statin Toxicity. Mechanistic Insights and Clinical Implications 17 Jan 2019 https://doi.org/10.1161/ CIRCRESAHA.118.312782Circulation Research. 2019;124:328 - 350.

6. Cannistraro RJ, Badi M, Eidelman BH, Dickson DW, Middlebrooks EH, Meschia JF. CNS small vessel disease: A clinical review. Neurology. 2019; 92(24):1146-1156. doi:10.1212/WNL.0000000000 007654.

7. Guo Y, Li Y, Liu X, et al. Assessing the effectiveness of statin therapy for alleviating cerebral small vessel disease progression in people $\geqslant 75$ years of age. BMC Geriatr. 2020; 20(1): 292. Published 2020 Aug 17. doi:10.1186/s12877-020-01682-w.

8. Zhang $X$, Wen J, Zhang Z. Statins use and risk of dementia: A dose-response meta analysis.
Medicine (Baltimore). 2018; 97(30): e11304. doi:10. 1097/MD.0000000000011304.

9. Chang CF, Liou YS, Lin TK, et al. High exposure to statins decrease the risk of new-onset dementia: $A$ nationwide population-based longitudinal cohort study. Medicine (Baltimore). 2019;98(34):e16931.

10. Chen PY, Liu SK, Chen CL, Wu CS. Long-term statin use and dementia risk in Taiwan. J Geriatr Psychiatry Neurol. 2014; 27(3): 165-171. doi:10.1177/08919 88714522702.

11. Bettermann K, Arnold AM, Williamson J, et al. Statins, risk of dementia, and cognitive function: secondary analysis of the ginkgo evaluation of memory study. J Stroke Cerebrovasc Dis. 2012; 21(6): 436-444. doi:10.1016/j.jstrokecerebrovasdis. 2010.11.002.

12. Lin FC, Chuang YS, Hsieh HM, et al. Early Statin Use and the Progression of Alzheimer Disease: A Total Population-Based Case-Control Study. Medicine (Baltimore). 2015; 94(47): e2143. doi:10.1097/MD.0000000000002143.

13. Chou CY, Chou YC, Chou YJ, Yang YF, Huang N. Statin use and incident dementia: a nationwide cohort study of Taiwan. Int J Cardiol. 2014; 173(2): 305-310. doi:10.1016/ j.ijcard.2014.03.018.

14. Wu CK, Yang YH, Lin TT, et al. Statin use reduces the risk of dementia in elderly patients: a nationwide data survey and propensity analysis. J Intern Med. 2015; 277(3):343-352. doi:10.1111/joim.12262.

15. Wolozin B, Wang SW, Li NC, Lee A, Lee TA, Kazis LE. Simvastatin is associated with a reduced incidence of dementia and Parkinson's disease. BMC Med. 2007;5:20. Published 2007 Jul 19. doi:10.1186/1741-7015-5-20.

16. Horsdal HT, Olesen AV, Gasse C, Sørensen HT, Green RC, Johnsen SP. Use of statins and risk of hospitalization with dementia: a Danish population based case-control study. Alzheimer Dis Assoc Disord. 2009; 23(1): 18-22. doi:10.1097/NAD. 0b013e318180f55b.

17. Reed B, Villeneuve S, Mack W, DeCarli C, Chui HC, Jagust $W$. Associations between serum cholesterol levels and cerebral amyloidosis. JAMA Neurol. 2014; 71(2):195-200. doi:10.1001/jamaneurol.2013. 5390.

18. Geifman N, Brinton RD, Kennedy RE, Schneider LS, Butte AJ. Evidence for benefit of statins to modify cognitive decline and risk in Alzheimer's disease. Alzheimers Res Ther. 2017;9(1):10. Published 2017 Feb 17. doi:10.1186/s13195-017-0237-y.

19. Guo Y, Li Y, Liu X, et al. Assessing the effectiveness of statin therapy for alleviating cerebral small vessel disease progression in people $\geqslant 75$ years of age. BMC Geriatr. 2020; 20(1): 292. Published 2020 Aug 17. doi:10.1186/s12877-020-01682-w. 
20. Shepherd J, Blauw GJ, Murphy MB, et al. Pravastatin in elderly individuals at risk of vascular disease (PROSPER): a randomised controlled trial. Lancet. 2002; 360(9346): 1623-1630. doi:10.1016/s 0140-6736(02)11600-x.

21. Wanamaker BL, Swiger KJ, Blumenthal RS, Martin SS. Cholesterol, statins, and dementia: what the cardiologist should know. Clin Cardiol. 2015; 38(4): 243-250. doi: 10.1002/clc.22361.

22. Hendrie HC, Hake A, Lane $\mathrm{K}$, et al. Statin Use, Incident Dementia and Alzheimer Disease in Elderly African Americans. Ethn Dis. 2015; 25(3): 345-354. Published 2015 Aug 7. doi:10.18865/ed.25.3.345. 